\title{
LIBRI RICEVUTI E RECENSIONI
}

\section{Libri ricevuti.}

T. DUNCAN: Electronics and Nuclear Physics. John Murray, London, 1966; p. $\mathrm{x}-286$; s.i.p.

L. TIsza: Generatized Thermodynamics. The M.I.T. Press, Cambridge, Mass. and London, England, 1966; p. XII-384; \$10.00.

H. E. FetTis and J. C. CASLIN: An Extended Table of Zeros of Oross Produets of Bessel Functions. Aerospace Research Laboratories, U. S. Air Force, WrightPatterson Air Force Base, Ohio, 1966; p. v-126; s.i.p.

\section{Recensioni.}

J. D. BJORKen e S. D. DReLLL - Relativistic Quantum Fields. MeGraw-Hill Book Co., New York, St. Louis, San Francisco, Toronto, London, Sydnej, 1965; p. XIV-396; \$12.50.

Relativistio Quantum Fields fa seguito al libro Relativistic Quantum Meohanics degli stessi antori, e con esso costituisce un'opera in due volumi dedicata alla teoria dei campi quantizzati. Nonostante quest'opera fosse stata inizialmente concepita dagli autori come un unico libro, Relativistic Quantum Fields risulta largamente indipendente dal volume precedente, rendendone così possibile lo studio anche a chi non aresse aruto occasione di conoscere la prima parte.

Gli argomenti trattati sono, oltre al formalismo canonico ed alla quantizzazione dei campi liberi, un capitolo dedicato ai campi in interazione e alle loro simmetrie, il formalismo LSZ, la teoria delle perturbazioni, proprietà di analiticità dei grafici di Feynman, relazioni di dispersione, ed infine un capitolo dedicato alla teoria della rinormalizzazione.

Le principali lacune di questo libro sono elencate dagli stessi autori nella prefazione: sostanzialmente si può notare che poco spazio è dedicato alle applicazioni ed ai problemi più avanzati della teoria dei campi, come pure ai (non molti) risultati raggiunti dalle teorie assiomatiche. Queste lacune, più che costituire difetti del libro, ne precisano i limiti entro cui esso mantiene la sua validità. Semmai è da notare che certi problemi, come quelli che fanno capo al teorema di Haag, anche in un testo senza eccessive pretese di rigore e sensibilità per le questioni più delicate, meriterebbero qualcosa di più che una nota a piè di pagina, che tra l'altro si può prestare ad una interpretazione errata.

Nonostante queste riserve, il libro nel complesso è buono e ritengo che possa 